\title{
ON CRITERIA OF DEFINABILITY
}

\author{
WALTER FELSCHER
}

A class $\mathbf{K}$ of relational systems is $A C_{\delta}$ if there exists a set $S$ of sentences (of the language determined by the type of relational systems in $\mathbf{K}$ ) such that $\mathbf{K}$ consists precisely of the models of $S: \mathbf{K}$ $=\operatorname{Mod}(S)$. The class $\mathrm{K}$ is $A C$ if, moreover, a finite set $S$ can be chosen here. If $\mathbf{L}$ is a class of relational systems, a class $\mathbf{K} \subseteq \mathbf{L}$ is $A C_{\delta}(\mathbf{L})$ or $A C(\mathbf{L})$ if there exists a class $\mathbf{K}^{\prime}$ such that $\mathbf{K}=\mathbf{K}^{\prime} \cap \mathbf{L}$ and $\mathrm{K}^{\prime}$ is $A C_{\delta}$ or $A C$ respectively. Let $\Pi, \Omega, \varepsilon \varepsilon, g$ be the operations of taking ultraproducts, ultralimits, elementary subsystems, and isomorphic images. Kochen [4] has shown

Theorem 1. If $\mathbf{K} \subseteq \mathbf{L}, \mathbf{K}$ is closed under $\Pi, \Omega, \mathfrak{g}$ and $\mathbf{L}-\mathbf{K}$ is closed under $\Omega$, then $\mathbf{K}$ is $A C_{\delta}(\mathbf{L})$. If, moreover, $\mathbf{L}-\mathbf{K}$ is closed under $\Pi$, then $\mathbf{K}$ is $A C(\mathbf{L})$.

Kochen's proof is based on his earlier characterization of classes $A C$ and $A C_{\delta}$ (Kochen [3, Theorem 11.6]), which follows from the present theorem by taking $\mathbf{L}$ to be the class of all relational systems of the type under consideration. Now Kochen's proof of that characterization depended on a thorough analysis of the relationship between ultralimits and prenex normal forms. It is a first purpose of this note to give a new proof of Theorem 1, based solely on the following facts:

(1) If $\left\langle A_{i} \mid i \in I\right\rangle,\left\langle B_{i} \mid i \in I\right\rangle$ are sequences of relational systems and $A_{i} \equiv B_{i} \quad\left(A_{i}\right.$ elementarily equivalent to $\left.B_{i}\right)$ for every $i \in I$, then $\Pi_{D}\left\langle A_{i} \mid i \in I\right\rangle \equiv \Pi_{D}\left\langle B_{i} \mid i \in I\right\rangle$ for every ultrafilter $D$ on $I$ (FrayneMorel-Scott [1, Corollary 2.4]).

(2) If $A \equiv B$, then $A$ and $B$ have isomorphic ultralimits (Kochen [3, Theorem 9.3]).

(3) A class $\mathbf{K}$ is $A C_{\delta}$ if and only if $\mathbf{K}$ is closed under $\Pi$ and $\varepsilon$ (elementary equivalence) (Frayne-Morel-Scott [1, Theorem 2.13]). It should be noted that both (2) and (3) follow rather directly from Frayne's lemma.

(4) If $A \equiv B$, then $B$ is isomorphic to an elementary subsystem of a suitable ultrapower of $A$ (Frayne-Morel-Scott [1, Theorem 2.12], Kochen [3, Lemma 9.1]).

It is well known that Kochen's characterization of classes $A C_{\delta}$ is an easy consequence of (2) and (3).

For a proof of Theorem 1 , assume $\mathbf{K} \subseteq \mathbf{L}$ and define $\mathbf{C}(\mathbf{K})$ by $A \in \mathbf{C}(\mathbf{K})$ if there exists $A^{\prime} \in \mathbf{K}$ and $A^{\prime} \equiv A$; define $\mathbf{C}(\mathbf{L}-\mathbf{K})$ sim-

Received by the editors April 21, 1967. 
ilarly. If $\mathbf{K}$ is closed under $\Pi$, it follows from (1) that $\mathbf{C}(\mathbf{K})$ is closed under $\Pi$. Hence $\mathbf{C}(\mathbf{K})$ is $A C_{\delta}$ by (3). If both $\mathbf{K}$ and $\mathbf{L}-\mathbf{K}$ are closed under $\Omega$ and $\mathbf{X}$ is closed under $\mathscr{g}$, it follows from (2) that $\mathbf{C}(\mathbf{K})$ and $\mathbf{C}(\mathbf{L}-\mathbf{K})$ are disjoint. Hence $\mathbf{C}(\mathbf{K}) \cap \mathbf{L}=\mathbf{K}$, i.e. $\mathbf{K}$ is $A C_{\delta}(\mathbf{L})$. If, moreover, $\mathbf{L}-\mathbf{K}$ is closed under $\Pi$, also $\mathbf{C}(\mathbf{L}-\mathbf{K})$ is $A C_{\delta}$ by symmetry. It can be assumed now that neither $\mathbf{K}$ nor $\mathbf{L}-\mathbf{K}$ are empty. If $\mathrm{Th}(\mathbf{K})$ and $\operatorname{Th}(\mathbf{L}-\mathbf{K})$ are the sets of sentences true in $\mathbf{K}$ and $\mathbf{L}-\mathbf{K}$ respectively, then $\mathbf{C}(\mathbf{K}) \cap \mathbf{C}(\mathbf{L}-\mathbf{K})=0$ shows that $\operatorname{Th}(\mathbf{K}) \cup \operatorname{Th}(\mathbf{L}-\mathbf{K})$ is inconsistent, while $\operatorname{Th}(\mathbf{K})$ and $\operatorname{Th}(\mathbf{L}-\mathbf{K})$ alone are consistent. Hence there exist finite and nonempty subsets $S_{0} \subseteq \mathrm{Th}(\mathbf{K}), S_{1} \subseteq \mathrm{Th}(\mathbf{L}-\mathbf{K})$ such that $S_{0} \cup S_{1}$ is inconsistent, whence $\operatorname{Mod}\left(S_{0}\right) \cap \operatorname{Mod}\left(S_{1}\right)=0$. Therefore $\operatorname{Mod}\left(S_{0}\right) \cap \mathbf{L}=\mathbf{K}$, i.e. $\mathbf{K}$ is $A C(\mathbf{L})$.

An even simpler argument, making use of (4) instead of (2), yields

THEOREM 2. If $\mathbf{K} \subseteq \mathbf{L}, \mathbf{L}$ is closed under $\mathfrak{g}$ and $\mathbf{K}$ is closed under $\Pi$, $g, \varepsilon z \backslash \mathbf{L}$, then $\mathbf{K}$ is $A C_{\delta}(\mathbf{L})$. If, moreover, $\mathbf{L}-\mathbf{K}$ is closed under $\Pi$, then $\mathrm{K}$ is $A C(\mathbf{L})$.

Kochen ([3, Theorem 12.1], [4, Theorem 3]) has used Theorem 1 in order to give a mathematical characterization of definable model functions, of which Beth's theorem on definability is an immediate consequence. Here a model function $U$ on a class $\mathbf{L}$ of relational systems assigns to every $A \in \mathbf{L}$ a relational system $U(A)=\left\langle A, R_{A}\right\rangle$, having one additional new relation $R_{A}$ of a fixed arity. $U$ is definable with respect to $L$ if there exists a formula of the language determined by $\mathrm{L}$ which, for every $A \in \mathrm{L}$, defines the relation $R_{A}$ in terms of $A$. Now Theorem 2 can be applied in order to obtain

TheORem 3. Let $U$ be a model function on $\mathrm{L}$, and let $\mathrm{L}$ be closed under $g$ and $\Pi$. $U$ is definable with respect to $\mathrm{L}$ if and only if $U$ commutes with the operations $\Pi, \mathfrak{g}$ and $\varepsilon 8$.

The proof is essentially that of Kochen's Theorem 12.1 in [3]. Namely, let the relations $R_{A}$ be $n$-ary. Let $L^{\prime}$ be the class of all relational systems $\langle A, \alpha\rangle$, where $A \in \mathrm{L}$ and $\alpha$ is a sequence of $n$ elements of the set $s(A)$ underlying $A ; \mathbf{L}^{\prime}$ then is described by a type that extends the type of $\mathrm{L}$ by $n$ new constants. Let $\mathrm{K}$ be the class of all $\langle A, \alpha\rangle$ in $\mathbf{L}^{\prime}$ such that $\alpha \in R_{A}$. $U$ will be definable with respect to $\mathrm{L}$ if and only if $\mathbf{K}$ is $A C\left(\mathbf{L}^{\prime}\right)$. Now with $\mathbf{L}$, also $\mathbf{L}^{\prime}$ is closed under $g$. It follows from Kochen's proof that $U$ commutes with $\Pi$ and $g$ if and only if $\mathbf{K}$ and $L^{\prime}-K$ are closed under $\Pi$ and $g$. Further, if $U$ is definable then it commutes with $8 \&$. Assume now that $U$ commutes with $\varepsilon \&$ and let $\langle B, \beta\rangle \in \mathbf{L}^{\prime}$ be an elementary subsystem of $\langle A, \alpha\rangle \in \mathbf{K}$. Then $\beta=\alpha$, and $B$ is an elementary subsystem of $A$. Therefore, 
$\left\langle B, R_{B}\right\rangle$ is an elementary subsystem of $\left\langle A, R_{A}\right\rangle$, and in particular $R_{B}=s(B)^{n} \cap R_{A}$. But then $\beta=\alpha$ and $\alpha \in R_{A}$ implies $\beta \in R_{B}$, i.e. $\langle B, \beta\rangle \in \mathbf{K}$.

Theorem 3 has applications in Hoehnke's work [2] on the mathematical characterization of definable maps between classes of relational systems.

It follows from well-known results of Keisler's that sufficient belief in $\mathrm{GCH}$ would enable us to omit any assumptions concerning 88 in Theorem 2 and Theorem 3.

\section{REFERENCES}

1. T. Frayne, A. C. Morel and D. S. Scott, Reduced direct products, Fund. Math. 51 (1962), 195-228.

2. H. J. Hoehnke, Über Modellkorrespondenzen, (to appear).

3. S. Kochen; Ultraproducts in the theory of models, Ann. of Math. 74 (1961), 221-261.

4. - "Topics in the theory of definition," pp. 170-176, in The theory of models, Amsterdam, 1965.

Mathematisches Institut der Universität Freiburg, West Germany 